\title{
Cloud Requirement and Cloud Analysis
}

\author{
Dr. Gurdev Singh \\ Professor \& Head \\ Adesh Institute of Engineering \\ \& Technology, Faridkot, \\ Punjab -151203, India
}

\author{
Harmandeep Singh \\ Assistant Professor \\ S.G.A.D.College \\ Khadur Sahib-143117
}

\author{
Amandeep Singh \\ Adesh Institute of Engineering \\ \& Technology, \\ Faridkot,Punjab-151203, India
}

\begin{abstract}
Cloud computing is model which uses combine concept of "software-as-a-service" and "utility computing",provide convenient and on-demand services to requested end users. Cloud Engineering is a discipline that uses combination of the business and technology thinking for purposes of engineering a cloud. The structure and principles of cloud engineering plays an important role for the engineering of usable and economic cloud. The cloud development life cycle (CDLC) is the one of the component in structure of cloud engineering with Base, Tools \& Techniques and Management components. CDLC is the iterated life cycle model for development, deployment and delivery of cloud. CDLC consists of six phases that are flexible, isolated executable and having feedback. The work on this paper focuses on the Understanding Requirement and Analysis phase of the CDLC. This paper lists and discusses the basic, content and operational requirements for the engineering of economic cloud. It also discuses feasibility checks for the requirements. Analysis part of this phase does the analysis of their cloud computing solution with another available computing solution such as grid computing, volunteer computing, utility computing, autonomic computing, clientserver model and peer to peer model; and also does cloud planning for cloud implementation phase.
\end{abstract}

\section{General Terms}

Cloud Computing, Cloud Engineering

\section{Keywords}

Cloud element, Open client, Service Level Agreement, Cloud Requirements, Cloud Analysis

\section{INTRODUCTION}

Cloud computing is a model for enabling convenient, ondemand network access to a shared pool of configurable computing resources that can be rapidly provisioned and released with minimal management effort [1] [2]. In simple words, the Cloud Computing is combination of a technology, platform, hosting provider, storage services provider on the Internet [3]. The aim of the cloud computing is to provide scalable and inexpensive on-demand computing infrastructures with good quality of service levels [4] [5]. To successful implementation of cloud computing solution, a cloud engineering discipline is used. Cloud Engineering is a discipline that uses both the business and technology thinking for purposes of engineering a cloud [6]. For engineering a cloud, there is very important to follow a model. Cloud development life cycle (CDLC)is the one of the available component model used in the structure of the cloud engineering[7].CDLC is the iterated, linear organized, isolated executable and flexible life cycle model for the systematic and scientifically development, deployment and delivery of cloud. CDLC contains six phase: Understand Requirement and Analysis; Architect; Implementation and Integration; Quality Assurance and Verification; Deploy, Testing and Improvement; Monitor, Migrate and Audit.The phase Architect; Quality Assurance and Verification; Deploy, Testing and Improvement; Monitor, Migrate and Audit have given its feedback to Understanding Requirement and Analysis phase [7].

Understanding requirement and analysis is the first step leading towards the engineering a usable and economic cloud. This phase evaluates and understands the needs of end user and network; and analysis it from the prospective of feasibility of solution of a network problem. The Architect phase is related to development of on-premise resource, cloud resources, cloud services, cloud middleware, software components, data server location used for engineering a cloud. The major building blocks of architecture are reference architecture, technical architecture and deployment and operational architecture. Implementation and integration phase is related to actual creation, enablement of the cloud solution, cloud file system while considering implementation issues. This phase has ability to communicate with both source and target systems; and extracting and publishing the data from the source to target system while considering integration patterns. The Quality assurance and verification is related to ensure the quality and web service of cloud network; and verify the performance, reliability, availability, elasticity and security of cloud network at the service level against specification, agreement and requirement [7].

Deploy, Testing and Improvement phase uses various platform service providers that significant reduces the deployment cost of the application by pre-building and preconfiguring a stack of application infrastructure. For this the developer chooses one of the available six deployment models. Cloud Testing test the functionality of various cloud services provided that comes between the cloud network and end user. Cloud improvement is the enhancing the entire development, deployment and testing process of cloud environment. Monitor, Migrate and Audit phase of continues monitors the cloud environment so that it can measure the performance of the cloud system. It also discusses the total cost and value that a user bears while evolving and moving to cloud approaches from traditional service-oriented environments and also integration with existing systems. Cloud audit is the audit of cloud for compliance, regulation, security, governance and risk of cloud [7]. 
In this paper, research focus only at the Understanding Requirement and Analysis phase of CDLC. This phase of cloud development life cycle model understands and evaluates the needs of end user. This is done by knowing the complaints from end user, network solution, management and customers of the current system. When this complaint is seriously studied, they highlight some new requirements and deficiencies. Then it searches for the tentative cloud computing solution and defines cloud computing strategy which meets requirement and minimize deficiencies. It also accesses that cloud computing solution on the various factors such as computing cost, scalability, organizational agility and benefits. The Analysis part of the life cycle phase perform the analysis of their cloud computing solution with another available computing solution such as grid computing, volunteer computing, utility computing, autonomic computing, dynamic datacenter alliance solution [8].It also consider various aspects of adopting cloud computing solution such as privacy, security, immaturity of organization, risk involvement, reliability, performance and portability. A simple six steps activity of the understanding requirement and analysis phase is summarized in figure 1 .

1. Know the Basic, Content and Operational Requir ements of end use

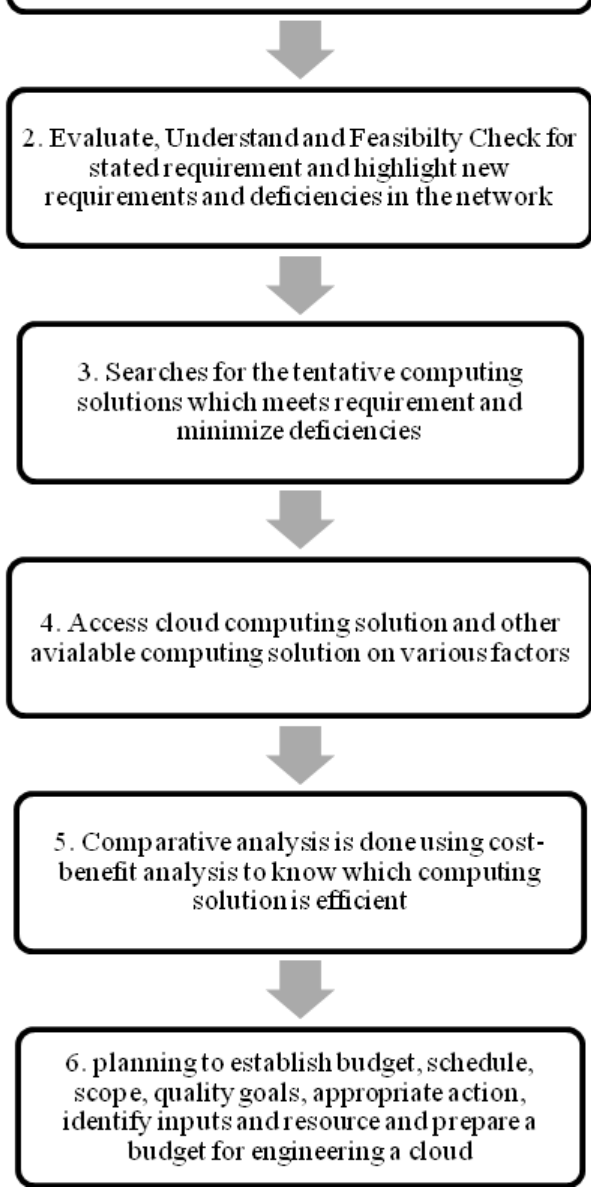

Figure 1: Step by step activities of understanding requirement and analysis phase
The first two activities shown in figure 1 are done in the requirement part and last four activities done in analysis part of the phase. Each activity is discussed and explored in the following sections. A detailed discussion on the cloud requirements and cloud analysis is also done in section 2 and section 3 .

\section{CLOUD REQUIREMENTS}

Knowing the requirement for a cloud is an art and requires lots of skills. To know the requirement, first of all, identify and define the basic element for a specific cloud system with their overall hierarchy in the system. After knowing the element, translate the user's desire capabilities and functions into proposed cloud computing system. There is need of the appropriate method for understanding what the customer wants, analyzing their need, assessing feasibility, negotiating a reasonable solution, specifying the solution unambiguously, validating the specification and managing the requirement as they are transformed into an operational system.

Requirement in cloud can be categorized in three parts: Basic, Content and Operational requirements. These cloud requirements are understood and well depicted by figure 2 .

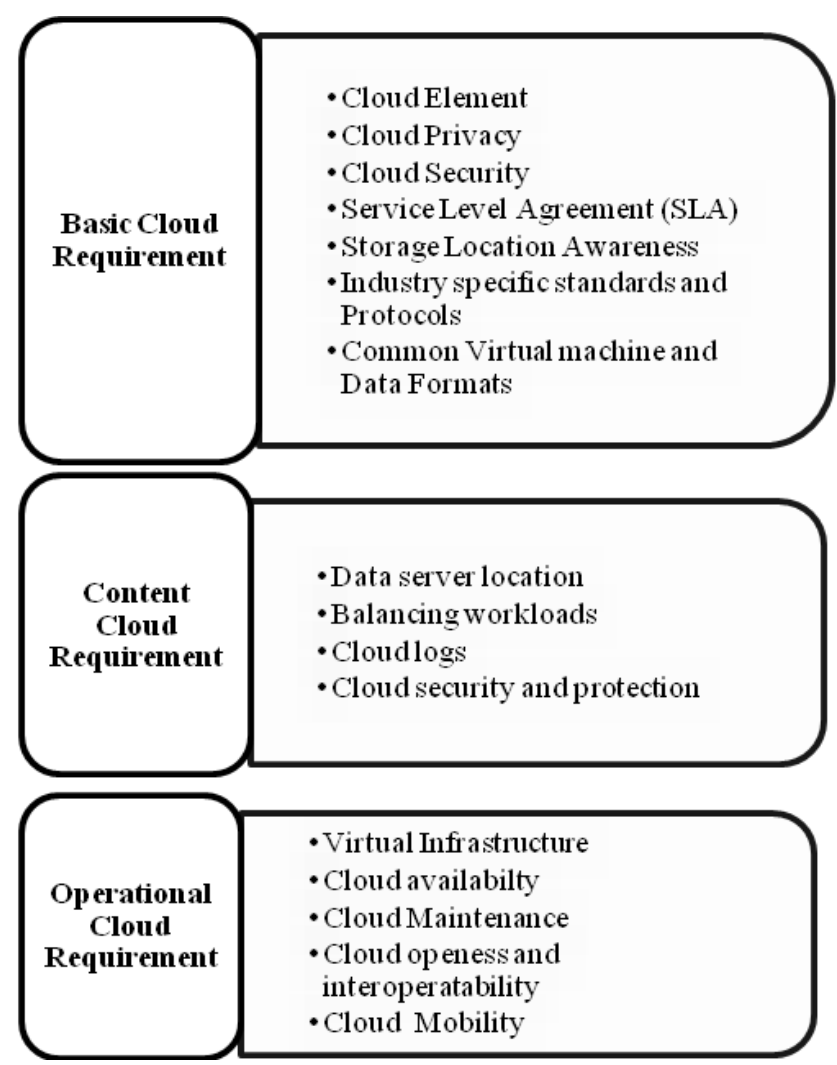

Figure 2: Cloud Requirements

A detailed discussion on three types of cloud requirements are done one by one in the following subsections.

\subsection{Basic Requirement}

The basic requirement for the development of cloud are cloud element, open client, privacy, security, service level agreement (SLA), location awareness, metering and monitoring services; industry specific standards, industry 
specific protocols, common virtual machine formats, data formats and API. The cloud element is aidentity which recognize and authenticates the end user. The open client is a computer hardware, operating system, web browser or Software that relies on cloud computing for application deliveryand cloud servicewithout the any use of platform.Cloud privacy is important requirement as the service provider at any point may access the data, store the data on the cloud and communication is done between end user and service provider. They could accidentally or deliberately alter, insert or delete some data on the cloud. Cloud security is implemented through cryptography, public key infrastructure (PKI), multiple cloud providers, standard API's, virtual machine support and legal support. Cloud security considers some issues while implementation process such as sensitive data access, data segregation, privacy, bug exploitation, recovery, accountability, malicious insiders, management console security, account control, and multitenancy.

Service level agreement (SLA) is the agreement between consumers and providers which ensures minimum service to the end user due to dynamic nature of cloud, quality of service (QoS), cloud consumer trust. The Service level agreement is much simpler for enterprise than individual businesses. Cloud provider must be clear about their services and gives guarantees of service which they provide at service, customer, multilevel and corporate level. Storage location awareness is the location of data servers depending on the kind of data the enterprise is managing on the user's behalf such that there is no legal restriction on the location of the physical server. Metering and Monitoring cloud service means the all clouds service must be metered and monitored for cost control, chargeback and for provisioning of a service. Industry specific standards and protocols are the application standards, common virtual machine formats, data formats and API.There are some extra basic requirements for enterprises for the adaption of the cloud services such as availability, data portability, application portability, data security, manageability, elasticity and federated System.

\subsection{Operational and ContentRequirement}

VMware's cloud blueprint [9] gives operational and content requirements for the engineering of quality IaaS cloud. A cloud must be built on a pooled and virtual infrastructure such as the CPU, memory, storage device and networking resource. A cloud should provide application, service and consumer mobility between different clouds. The use of existing consumer tools for performing the migration task of workloads to cloud or from a cloud is highly desirable. A cloudshould be open and interoperable so that consumer consumes cloud resources over open and internet standard protocols. A cloud consumer should only pay for resources they consume. A cloud should be a secure and trusted location for running cloud consumer workloads. A cloud consumer should have the option and the ability to protect their cloud-based workloads from data loss. A cloud consumer must not be responsible for the maintenance of the shared infrastructure. A cloud should be available to run with high availability workloads and any faults occurring in a cloud infrastructure should be transparent to a cloud consumer. A cloud should have a built-in orchestration framework that enables the quick scaling of infrastructure for cloud consumers. A cloud consumer should have access to detailed logs from a cloud environment and save them for future use. These logs should show only the entries pertinent to a cloud consumer's workloads. A cloud consumer should have a mechanism to balance the load between their workloads across a cloud infrastructure. A cloud consumershould have a mechanism to take snapshots of their workloads and save them for future use. There should be some way for the cloud provider to monitor capacity and performance of the environment and workloads so that the SLA can be ensure. A cloud consumer and provider should have a location for storing and using their own common set of images templates in catalog. After stated requirement, a feasibility check is essential to do for requirement.

\subsection{Feasibility Check}

A feasibility check is also done to ensure the required hardware, software, web service, money and storage area is available for engineering of aeconomic and quality cloud based upon the requirement known. Also check the proposed cloud in the light of its workability, meeting the user's requirements, effective use of resources and the cost effectiveness of cloud system.

\section{CLOUD ANALYSIS}

After understanding, analyzing, validating and specifying requirement, the next step to engineer a cloud is the cloud analysis. The cloud analysis starts with a high-level problem statement. The problem domain and the environment are understood and then model to know the system behavior and constraints on the various cloud inputs and outputs. The purpose of this activity is to obtain a thorough understanding of what the cloud computing system needs to provide services to end users and this is happen by face to face discussion of client and users about work, environment, and their needs. After clarifications of the understanding, this phase leads to specifying the cloud requirements which address the issues such as representation, specification languages, and tools. The basic goal of the analysis phase is to understand problem domain, that is, what exactly is desired from a cloud and what are various constrains on the solution of cloud computing problem.Cloud analysis can be done by two ways: formal and informal way. To know the constraints on cloud solution, many techniques are used such as divide and conquer;state and projection techniques.

\subsection{Analysis of Computing Solution}

After cloud analysis, a analyst perform the analysis of cloud computing solution of a network problem to know the performance, benefits and cost incurred during use of cloud solution. This phase also do same type of analysis with another available computing solution of a network problem such as grid computing, volunteer computing, utility computing, autonomic computing, client-server model, peer to peer model and dynamic datacenter alliance solution [8]. 


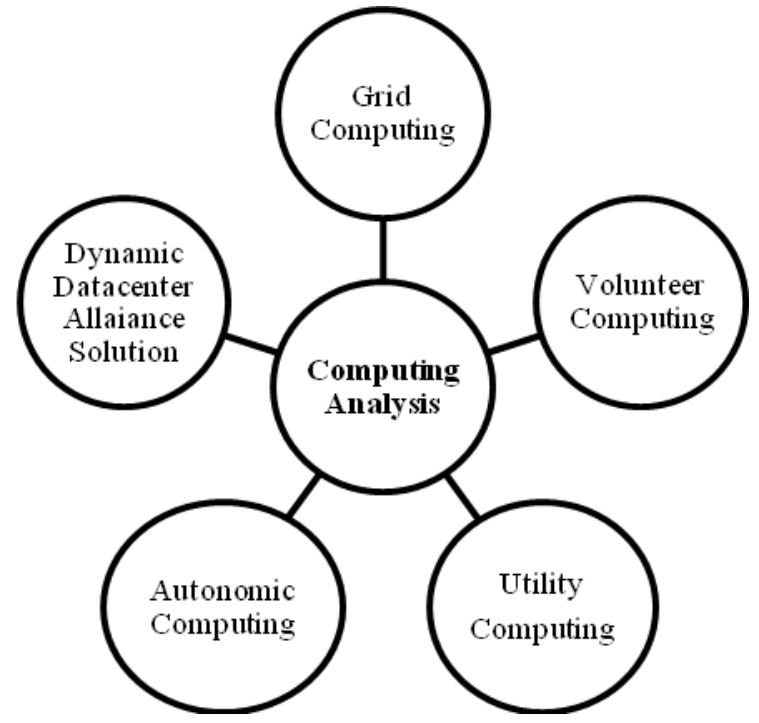

Fig 3: Cloud Computing Analysis

After performing analysis, a comparative analysis is also done to know which computing solution is efficient and best for implementation of solution of network problem. This analysis consider many issues while performing comparative analysis such as emerging trends, changing market conditions, competitive challenges, potential growth opportunities, cost-efficiency and on-demand capacity. To perform analysis, a cost-benefits analysis model is present which measure, compare and contrast the performance, costs and benefitsof various computing paradigms [10]. Every computing paradigm has its own performance, costs and benefits, which should be clear so that organization compare different available computing platform based upon factors such as efficiency, performance, platform engineering, application deployment, completion times, execution time, task redundancy, resource \& computation requirement, costs and benefits [10].

\subsection{Cloud Planning}

In the last, a planning should be done in order to establish cost, schedule, scope, quality goals, appropriate action, identify inputs, identify outputs, identify resources required, prepare a budget and draft plan for engineering a cloud. The input to the planning part is the requirements specification which is stated in first part of this paper. Cloud Planningactivity becomes essential to doso that implementation of a cloud will be efficient, effective and successfully on time.

\section{CONCLUSSION}

The cloud engineering is emerging as a new engineering discipline for the development of cloud computing solution.There is very important to follow a life cycle model for engineering a cloud. Cloud development life cycle is the one of the available model for this. Understanding requirement and analysis is the first step leading towards the engineering a usable and economic cloud. The understanding requirement and analysis phase of CDLC has listed the basic requirement for engineering of good quality cloud but these requirements are basic and minimum which require many refinement and exploration. In the future scope, the other phase of the cloud development life cycle such as Architect; Implementation and Integration; Quality Assurance and Verification; Deploy, Testing and Improvement; Monitor, Migrate and Audit can be explored and require lots of research.

\section{REFRENCES}

[1]http://searchvirtualdatacentre.techtarget.co.uk/news/15101 17/Community-cloud-Benefits-and-drawbacks

[2] Michael glas and paul Andres; "An Oracle white paper in enterprise architecture- achieving the cloud computing vision", CA-U.S.A, Oct 2010.

[3] Harjit Singh Lamba and Gurdev Singh, "Cloud Computing-Future Framework for e-management of NGO's", IJoAT, ISSN 0976-4860, Vol 2, No 3, Department Of Computer Science, Eternal University, Baru Sahib, HP, India, July 2011

[4] Dr. Gurdev Singh, ShanuSood, Amit Sharma, June 2011, "CM- Measurement Facets for Cloud Performance", IJCA, Volume 23 No.3, Lecturer, Computer science \& Engineering, Eternal University, Baru Sahib (India).

[5] Joachim Schaper, 2010, "Cloud Services", 4th IEEE International Conference on DEST, Germany.

[6] Stefan Tai, "Cloud Service Engineering", 18th IEEE International Workshops on Enabling Technologies, Karlsruhe Institute of Technology (KIT) and FZI ForschungszentrumInformatik, Karlsruhe, Germany, , 2009.

[7] Gurdev Singh, GauravGarg, Prince Jain, Harmandeep Singh, "The structure of cloud engineering", International Journal of Computer Applications 33(8):44-49, New York, USA, Nov 2011

[8] Prof. Dr. Andreas Polze, "A Comparative Analysis of Cloud Computing Environments", Operating Systems and Middleware, Hasso-Plattner-Institute for Software Engineering, University of Postdam, Germany, December 7, 2009.

[9] "VMware vCloud Requirements for a Cloud- Technical white paper", version 1.6, Inc3401, Hillview Ave Palo Alto, CA 94304, 2011.

[10] Derrick Kondo1, BahmanJavadi, Paul Malecot, Franck Cappello, David P Anderson, "Cost-Benefit Analysis of Cloud Computing versus Desktop Grids", INRIA, France, UC Berkeley, USA.

[11]http://cloudcomputing.ulitzer.com/node/920982

[12]http://www.applabs.com/html/TestingintheCloud_842.ht $\mathrm{ml}$

[13] Minqi Zhou, Rong Zhang, DadanZeng, WeiningQian, "Services in the Cloud Computing Era: A Survey", Software Engineering Institute, East China Normal University, Shanghai, China. NIICT, Kyoto, Japan.

[14]www.appliedcloudengineering.com 